\title{
Acute myocardial infarction after renal transplantation treated with percutaneous coronary intervention: a valuable lesson from a clinical case report
}

\author{
Yufan Yang, Yu Cao, Wei Huang, Weihong Jiang \\ Department of Cardiology of Third Xiangya Hospital attached to Central South University, Changsha, China \\ Correspondence to: Weihong Jiang, MD; Yu Cao, MD. Department of Cardiology of Third Xiangya Hospital attached to Central South University, \\ No. 138 Tongzi Po Road, Changsha 410013, China. Email: jwhxy3@sina.com; caoyu0811@csu.edu.cn.
}

\begin{abstract}
Acute myocardial infarction has been reported to be a common clinical complication after renal transplantation. This case report involves a retrospective review of the clinical management of a 56-year-old woman with a history of renal transplantation, who developed acute myocardial infarction and was treated with percutaneous coronary intervention and anti-coagulation therapy. After the treatment of percutaneous coronary intervention and anti-coagulation therapy, she was then readmitted to the hospital with upper gastrointestinal bleeding, renal calculus formation, and subsequent kidney injury. The unique of this case is that we encountered a patient who had acute myocardial infarction after renal transplantation with percutaneous coronary intervention treatment, with complications involving renal calculus formation and upper gastrointestinal bleeding. And we outline the risk factors of complications and the risk factors of antirejection and anti-coagulant drug therapies that had been prescribed to manage her renal transplantation and acute myocardial infarction. The lesson that we can learn from this clinical case report is that the followup management concerning patients with acute myocardial infarction after renal transplantation with percutaneous coronary intervention treatment is vital in the clinic and should be strengthened.
\end{abstract}

Keywords: Acute myocardial infarction (AMI); renal transplantation (RT); percutaneous coronary intervention; case report

Submitted Apr 16, 2020. Accepted for publication Oct 22, 2020.

doi: 10.21037/apm-20-962

View this article at: http://dx.doi.org/10.21037/apm-20-962

\section{Introduction}

Acute myocardial infarction (AMI) after renal transplantation (RT) has been reported to be a common clinical complication, and percutaneous coronary intervention (PCI) is known to be an effective treatment (1-3). During PCI treatment, patients are at risk of: (I) renal function damage due to contrast agent administration during PCI, especially for patients with RT; (II) injury to the gastric mucosa due to prednisone and immunosuppressant administration post-RT, and; (III) injury to the gastrointestinal (GI) mucosa due to dual-antiplatelet administration post-PCI that increases the risk of GI bleeding. Therefore, the management of patients with AMI post-RT is essential. This case report involved a patient who had an AMI post-RT and who had undergone PCI and developed complications with renal calculus formation and upper GI bleeding. We describe this patient's prognosis, complications and follow-up. Also, we discussed management to prevent complications in this clinical case, which is different to previous ones.

We present the following article in accordance with the CARE reporting checklist (available at http://dx.doi. org/10.21037/apm-20-962).

\section{Case presentation}

A 56-year-old woman with a 48-hour history of repeated chest pain was admitted to our hospital and was diagnosed 


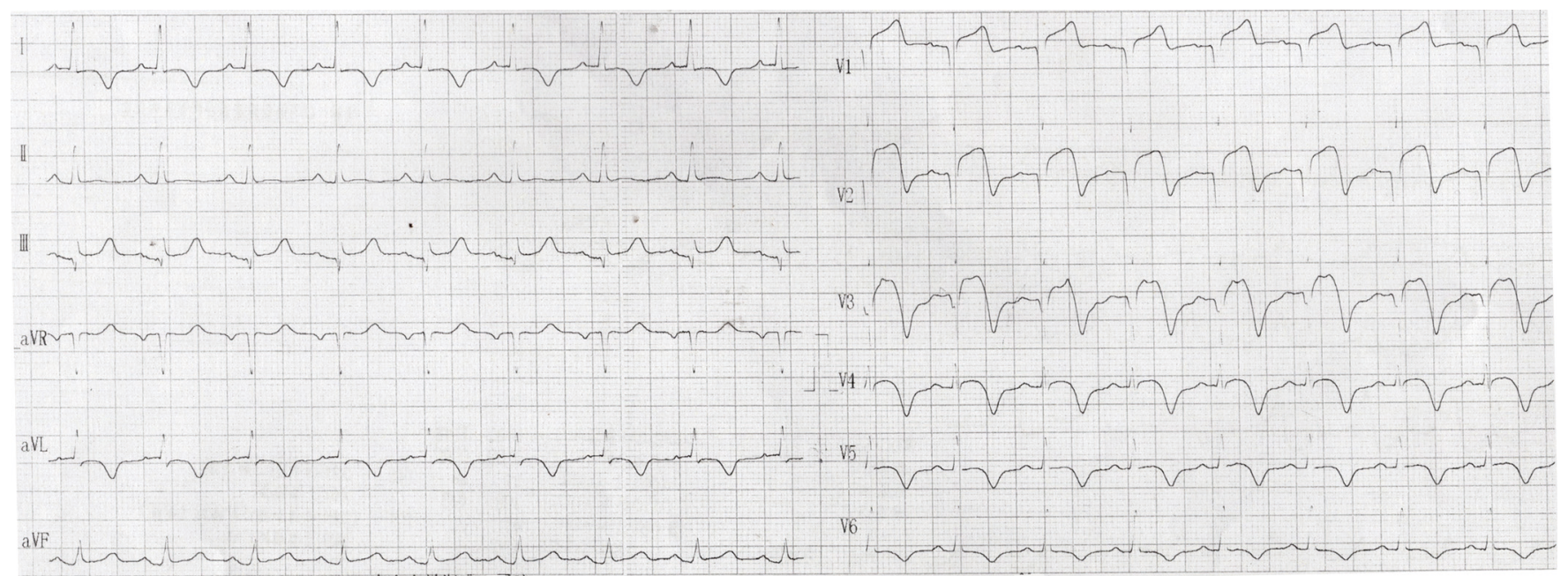

Figure 1 Patient electrocardiogram (ECG) characteristics showed ST elevation in leads $V_{1}-V_{4}$, indicating anterior wall myocardial infarction.

with AMI. Her past medical history included an 11-year history of hypertension, a 3-year history of end-stage renal disease, and a 2-month history of RT prior to AMI. Her family members had no myocardial infarction history. Electrocardiogram (ECG) results showed ST elevation in leads $\mathrm{V}_{1}-\mathrm{V}_{4}$ (Figure 1). Laboratory test results showed high-sensitivity troponin I $(3.624 \mathrm{ng} / \mathrm{mL})$, high amino terminal brain natriuretic peptide $(3,844.88 \mathrm{pg} / \mathrm{mL})$ levels, elevated creatine kinase (359 U/L), and the creatine kinase isoenzyme (64 U/L) levels. A coronary angiography was performed on August 20, 2018, revealing 3 branch stenosis lesions, with a SYNTAX score of 14 (Figure 2). She underwent PCI immediately following the coronary angiography. A PARTNER drug-eluting stent $(3.0 \times 15 \mathrm{~mm})$ was placed into the left anterior descending artery stenotic lesion (Figure 2). Dual antiplatelet therapy was started immediately (aspirin $300 \mathrm{mg}$ loading and $100 \mathrm{mg} /$ day maintenance; clopidogrel $300 \mathrm{mg}$ loading and $75 \mathrm{mg} /$ day maintenance) and she was discharged from hospital 6 days later.

One month after the PCI, she was readmitted to our hospital with oliguria and an increased plasma creatinine level. A color Doppler ultrasound showed renal calculus formation in the transplanted kidney. Laboratory test results showed a plasma uric acid (UA) level of $553 \mu \mathrm{mol} / \mathrm{L}$.

Four months following the PCI, she complained of a 24-hour history of black stools and hematemesis (volume, approximately $1,000 \mathrm{~mL}$ ) and she was readmitted to hospital for a third time. In the emergency room, she received an infusion of 6 units of concentrated red blood cells prior to admission. Gastroscopy results indicated that she had developed a duodenal bulbar ulcer. The timeline was drawn to conclude the whole procedure and critical information (Figure 3). The case report was approved by the Ethics Committee of the Third Xiangya Hospital of Central South University (NO.: 2020-S582). And all procedures performed in studies involving human participants were in accordance with the ethical standards of the institutional and/or national research committee(s) and with the Helsinki Declaration (as revised in 2013). Written informed consent was obtained from the patient.

Follow up: One year after PCI, we changed the antiplatelet therapy (only clopidogrel $75 \mathrm{mg}$ per day). And the patient never developed gastrointestinal bleeding and AMI.

\section{Discussion}

A review of this clinical case identified 3 characteristics: (I) AMI occurred 1 month after RT, (II) renal calculus developed 1 month after PCI, and; (III) upper gastrointestinal (GI) bleeding developed 4 months after PCI.

Risk factors for AMI post-RT are as follows. First, for patients regularly receiving prednisone (20 $\mathrm{mg}$ per day) and tacrolimus (2.5 mg per day) post-RT, plasma lipid levels increase and elevate the risk of AMI $(4,5)$. Pre-RT, the plasma low density-lipoprotein (LDL) level in our patient was $2.76 \mathrm{mmol} / \mathrm{L}$, total cholesterol was $5.09 \mathrm{mmol} / \mathrm{L}$, and the triglyceride level was $1.08 \mathrm{mmol} / \mathrm{L}$. While undergoing 

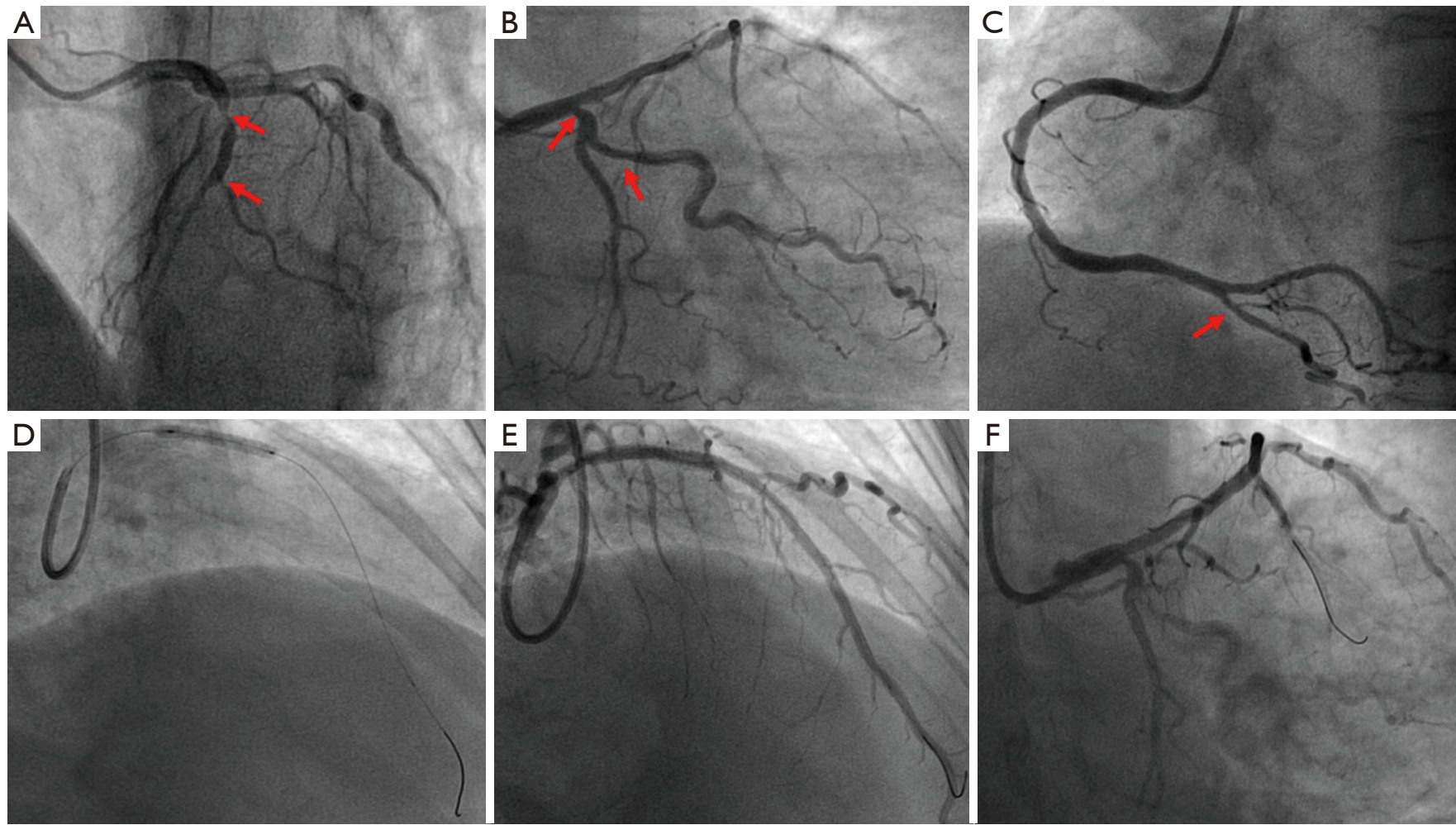

Figure 2 Angiographic findings: (A) Coronary angiography (CAG) revealed 95\% stenosis in the left anterior descending branch and $60 \%$ stenosis in the first diagonal branch. (B) CAG revealed $70 \%$ stenosis in the proximal circumflex branch and $60 \%$ stenosis in the first obtuse branch. (C) CAG revealed 50\% stenosis in the posterior descending branch. (D) A SPRINTER balloon $(2.0 \times 15 \mathrm{~mm})$ was pre-expanded in the target lesion. (E) A PARTNER stent $(3.0 \times 15 \mathrm{~mm})$ was successfully implanted in the left anterior descending branch. (F) CAG of the target lesion revascularization. Red arrow means the stenosis.

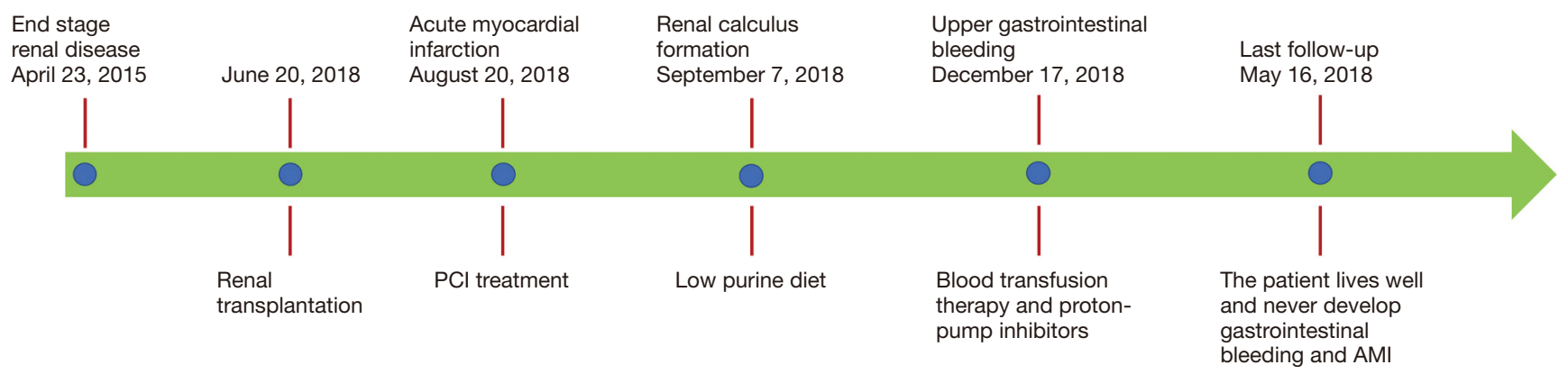

Figure 3 The timeline which was drawn to conclude the whole procedure and critical information. PCI, percutaneous coronary intervention.

regular prednisone and tacrolimus therapy post-RT for 1 month, she developed an AMI, and the plasma LDL increased to $3.18 \mathrm{mmol} / \mathrm{L}$, the total cholesterol level increased to $5.99 \mathrm{mmol} / \mathrm{L}$, and the triglyceride level was $2.54 \mathrm{mmol} / \mathrm{L}$. These results indicated that her plasma lipid levels had significantly increased 1 month post-RT and were closely linked to the administration of prednisone and tacrolimus. Second, the patient had a history of chronic hypertension, which is an established high-risk factor for AMI (6). Third, an elevated plasma UA level is a well- 
known high-risk factor for the incidence of AMI (7). During the AMI period, this patient had a plasma UA level of $320 \mu \mathrm{mol} / \mathrm{L}$, which remained at the higher end of the normal range after the PCI. The patient's laboratory findings indicated that the high plasma UA level was related to the post-RT AMI. As well as these risk factors being present, this patient was likely to have developed coronary artery lesions prior to her RT, even though she had no clinical manifestations. Therefore, strengthening the monitoring of risk factors for $\mathrm{AMI}$ in the perioperative RT period is needed to reduce the incidence of AMI.

A patient with RT is likely to develop elevated plasma UA levels, which can result in renal calculus formation in a transplanted kidney $(8,9)$. In this patient, no renal calculi were observed on color Doppler ultrasonography performed immediately post-RT. At one month post-PCI, color Doppler ultrasonographic findings showed renal calculus formation in the transplanted kidney. We evaluated the possible cause of renal calculus formation in this patient. Pre-RT, her plasma UA level was normal. Post-RT, the plasma UA level increased to $320 \mu \mathrm{mol} / \mathrm{L}$. At one month after PCI, she presented with clinical symptoms of oliguria. Her laboratory examination results also showed that the plasma creatinine level had increased, which indicated kidney injury. Her data revealed that her high plasma UA level was related to renal calculus formation in the transplanted kidney, that the transplanted kidney had been injured, and that the plasma creatinine level was high (10). The use of a contrast agent during the PCI would have increased the risk of kidney injury, the kidney injury, likely to be the contrast agent, was a risk factor in turn for elevated plasma UA and creatinine levels (11). However, this patient did not develop acute kidney injury (AKI) immediately post-PCI (plasma creatinine level, $66 \mu \mathrm{mol} / \mathrm{L}$ immediately post-PCI). At one month post-PCI, her plasma creatinine level had increased to $234 \mu \mathrm{mol} / \mathrm{L}$. Therefore, increased monitoring of plasma UA and creatinine levels as well as renal color Doppler ultrasound examinations should have been undertaken for several months post-PCI in this patient. Improved monitoring could have identified an elevated plasma UA level, which, in turn, have decreased the risk of renal calculus formation in the transplanted kidney and reduced the plasma creatinine level.

This patient developed upper GI bleeding 4 months after the PCI, and gastroscopy findings confirmed that she had developed a duodenal ulcer. During a retrospective review of her upper GI bleeding complications, we identified that she was also at risk in relation to the following two risk factors:
(I) patients receiving tacrolimus and prednisone postRT to inhibit rejection are at an increased risk of GI tract injury (12), and; (II) antiplatelet drugs such as clopidogrel and aspirin further increase the risk of GI bleeding. Therefore, to prevent the occurrence of upper GI bleeding due to ulceration in such patients, relevant laboratory examinations should be undertaken in the perioperative emergency PCI period to identify Helicobacter pylori infection. Moreover, additional drug-related gene detection should be undertaken in the perioperative emergency PCI period to determine the risk of aspirin-related bleeding, and patients should receive proton-pump inhibitors to prevent upper GI bleeding when taking aspirin and prednisone simultaneously. According to the guideline, we use the anti-coagulation therapy of aspirin $(300 \mathrm{mg}$ loading and $100 \mathrm{mg} /$ day maintenance) and clopidogrel (300 mg loading and $75 \mathrm{mg} /$ day maintenance) (13). Also, we can use indobufen instead of aspirin for antiplatelet therapy in the future because fewer adverse events and less gastrointestinal bleeding were seen with indobufen than with aspirin (14).

The strength of this clinical case is that we encountered an AMI post-RT patient treated with PCI and developed complications with renal calculus formation and upper GI bleeding. And we discussed the risk factors and management of these complications. It is different from previous ones. The limitation is that this is only one case. Thus, further multicenter studies conducted with larger sample sizes are needed to confirm the risk factors and management of these complications.

\section{Conclusions}

In conclusion, we encountered a patient who had AMI post-RT with PCI treatment, with complications involving renal calculus formation and upper GI bleeding. This case report indicates that follow-up management concerning patients with AMI post-RT with PCI treatment should be strengthened.

\section{Acknowledgments}

Funding: None.

\section{Footnote}

Reporting Checklist: The authors have completed the CARE reporting checklist. Available at http://dx.doi.org/10.21037/ apm-20-962 
Conflicts of Interest: All authors have completed the ICMJE uniform disclosure form (available at http://dx.doi. org/10.21037/apm-20-962). The authors have no conflicts of interest to declare. And all authors don't serve as current Editorial Team Members.

Ethical Statement: The authors are accountable for all aspects of the work in ensuring that questions related to the accuracy or integrity of any part of the work are appropriately investigated and resolved. The case report was approved by the Ethics Committee of the Third Xiangya Hospital of Central South University (NO.: 2020-S582). And all procedures performed in studies involving human participants were in accordance with the ethical standards of the institutional and/or national research committee(s) and with the Helsinki Declaration (as revised in 2013). Written informed consent was obtained from the patient.

Open Access Statement: This is an Open Access article distributed in accordance with the Creative Commons Attribution-NonCommercial-NoDerivs 4.0 International License (CC BY-NC-ND 4.0), which permits the noncommercial replication and distribution of the article with the strict proviso that no changes or edits are made and the original work is properly cited (including links to both the formal publication through the relevant DOI and the license). See: https://creativecommons.org/licenses/by-nc-nd/4.0/.

\section{References}

1. Kasiske BL, Maclean JR, Snyder JJ, et al. Acute myocardial Infarction and kidney transplantation. J Am Soc Nephrol 2006;17:900-7.

2. Rubenstein MH, Harrell LC, Sheynberg BV, et al. Are patients with renal failure good candidates for percutaneous coronary revascularization in the new device era? Circulation 2000;102:2966-72.

3. Stigant C, Izadnegahdar M, Levin A, et al. Outcomes after percutaneous coronary interventions in patients with CKD: improved outcome in the stenting era. Am J Kidney Dis 2005;45:1002-9.

4. Trikudanathan S, McMahon GT. Optimum management of glucocorticoid- treated patients. Nat Clin Pract Endocrinol Metab 2008;4:262-71.

5. Law YM, Yim R, Agatisa P, et al. Lipid profiles in pediatric thoracic transplant recipients are determined by their immunosuppressive regimens. J Heart Lung Transplant 2006;25:276-82.

6. Konstantinou K, Tsioufis C, Koumelli A, et al. Hypertension and patients with acute coronary syndrome: Putting blood pressure levels into perspective. J Clin Hypertens (Greenwich) 2019;21:1135-43.

7. Li L, Yi M, Xiao-Ming S, et al. Hyperuricemia is associated with short-term outcomes in elderly patients with acute myocardial infarction. Aging Clin Exp Res 2018;30:1211-5.

8. Haririan A, Metireddy M, Cangro C, et al. Association of Serum Uric Acid With Graft Survival After Kidney Transplantation: A Time-Varying Analysis. Am J Transplant 2011;11:1943-50.

9. Greif F, Dreznick Z, Jacob ET. Calculus in 16-YearOld Cadaveric Kidney Transplant: A Unique Case and Literature Review. Nephron 1990;55:423-8.

10. Viazzi F, Leoncini G, Rtto E, et al. Hyperuricemia and renal risk. High Blood Press Cardiovasc Prev 2014;21:189-94.

11. Chorin E, Ben-Assa E, Konigstein M, et al. Prevention of post procedural acute kidney injury in the catheterization laboratory in a real-world population. Int J Cardiol 2017;226:42-7.

12. Renner FC, Staak A, Bur Am Orde L, et al. Tacrolimus intoxication resolved by gastrointestinal bleeding: case report. Transplant Proc 2007;39:522-5.

13. Roffi M, Patrono C, Collet JP, et al. 2015 ESC Guidelines for the management of acute coronary syndromes in patients presenting without persistent ST-segment elevation: Task Force for the Management of Acute Coronary Syndromes in Patients Presenting without Persistent ST-Segment Elevation of the European Society of Cardiology (ESC). Eur Heart J 2016;37:267-315.

14. Bhana N, McClellan KJ. Indobufen: an updated review of its use in the management of atherothrombosis. Drugs Aging 2001;18:369-88.

Cite this article as: Yang Y, Cao Y, Huang W, Jiang W. Acute myocardial infarction after renal transplantation treated with percutaneous coronary intervention: a valuable lesson from a clinical case report. Ann Palliat Med 2021;10(6):7028-7032. doi: 10.21037/apm-20-962 